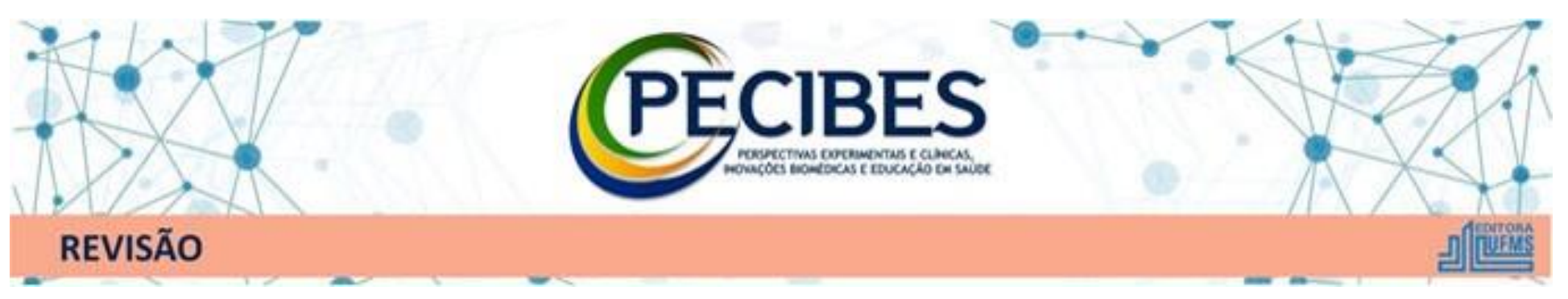

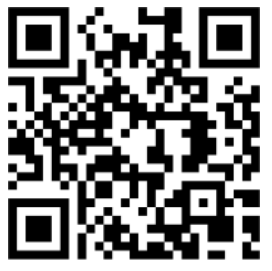

http://www.seer.ufms.br/index.php/p ecibes/index

*Autor correspondente: Jackelina de Lima Rodrigues. E-mail do autor: jacke.lina@hotmail.com

Palavras-chave: Plantas comestíveis. Dieta.

Alimentos e Nutrição. Cerrado.

Key-words: Plants edible.Diet. Food and Nutrition. Cerrado.

\section{Óleos, plantas e frutas do bioma cerrado utilizado na alimentação humana: revisão integrativa.}

Oils, plants and fruits belonging to the cerrado biome used in human feeding: integrative review.

Jackelina de Lima Rodrigues ${ }^{1}$, Marcos Antonio Ferreira Júnior ${ }^{1}$, Letícia Pinto Manvailer ${ }^{1}$, Mayk Penze Cardoso ${ }^{1}$, Oleci Pereira Frota ${ }^{1}$.

${ }^{1}$ Universidade Federal de Mato Grosso do Sul-UFMS

Resumo

Objetivou-se identificar os óleos, plantas e frutos pertencentes ao bioma cerrado brasileiro que apresentam potencial nutricional para uso na alimentação humana. O estudo trata de uma revisão integrativa de literatura e segue um rigor metodológico que contempla seis etapas, a saber: 1) Identificação da questão norteadora, 2) Seleção das fontes de pesquisa, 3) Seleção dos estudos relevantes ao considerar os critérios de inclusão e exclusão, 4) Extração dos dados por meio da síntese qualitativa, 5) Mapeamento dos resultados, com inclusão de tabelas e/ou gráficos na análise temática qualitativa, e 6) Divulgação dos resultados. A estratégia de busca ocorreu a partir do cruzamento de descritores controlados nas seguintes bases de dados: Biblioteca Virtual de Saúde (BVS), Science Direct e Cumulative Index of Nursing and Allied Health (CINAHL) with full text (EBSCO). Foram encontrados inicialmente um total de 13.489 estudos, que após a aplicação dos respectivos filtros com critérios de inclusão e exclusão, além da análise de qualidade metodológica, com obtenção de uma amostra final de seis artigos (n=6). As espécies Pyrostegiavenusta (Ker Gawl.); Dipteryx alata (Vog); Acrocomia aculeata; Dipteryxalata Vog. e Mauritia flexuosa L.f. apresentaram propriedades nutricionais e medicinais com potencial para auxiliar na suplementação dietética e assim gerar efeitos benéficos ao organismo humano. Sugere-se que mais estudos e ensaios clínicos sejam realizados ao considerar também a citotoxicidade de cada espécie, de forma a explorar ainda mais o potencial nutricional de óleos, plantas e frutos nativos do bioma cerrado brasileiro.

Abstract

The aim was to identify the oils, plants and fruits belonging to the Brazilian Cerrado biome that have nutritional potential for use in human food. The study deals with an integrative literature review and follows a methodological rigor that includes six steps, namely: 1) Identification of the guiding question, 2) Selection of research sources, 3) Selection of relevant studies considering the inclusion criteria and exclusion, 4) Data extraction through qualitative synthesis, 5) Mapping of results, including tables and / or graphs in the qualitative thematic analysis, and 6) Dissemination of results. The search strategy occurred from the crossing of controlled descriptors in the following databases: Virtual Health Library (VHL), Science Direct and Cumulative Index of Nursing and Allied Health (CINAHL) with full text (EBSCO). A total of 13.489 studies were initially found, which after applying the respective filters with inclusion and exclusion criteria, besides the methodological quality analysis, obtaining a final sample of six articles $(n=6)$. The species Pyrostegiavenusta (Ker Gawl.); Dipteryx alata (Vog); Aculeat acrocomia; Dipteryxalata Vog. and Mauritia flexuosa L.f. presented nutritional and medicinal properties with potential to assist in dietary supplementation and thus generate beneficial effects on the human organism. Further studies and clinical trials are suggested by considering also the cytotoxicity of each species, in order to further explore the nutritional potential of oils, plants and fruits native to the Brazilian cerrado biome. 


\section{Introdução}

O cerrado é um bioma que possui uma grande diversidade florística, com variedades de espécies frutíferas ricas em nutrientes distintos e características sensoriais peculiares, muitas vezes utilizadas na alimentação humana. Essas espécies frutíferas constituem fontes importantes de fibras, proteínas, minerais, ácidos saturados e insaturados presentes nas polpas e sementes (ABRAMOVAY, 1999).

O aumento contínuo no consumo de frutas tem sido uma importante tendência desta década. Componentes como fibras vitaminas, minerais e antioxidantes caracterizam a função diferenciada que as frutas exercem sobre $o$ funcionamento do organismo. Alguns compostos bioativos que dão aos frutos um caráter funcional desempenham um importante potencial de proteção e prevenção a algumas doenças causadas pelo estresse oxidativo, que ocorre em função da existência de um desequilíbrio entre compostos oxidantes e antioxidantes e gera uma quantidade de radicais livres maior do que a capacidade de remoção desses a partir de enzimas específicas, ocasionando diversos processos crônicos degenerativos, como por exemplo, as doenças cardiovasculares (BARBOSA et.al, 2010; SLOAN, 1999; KAUR; KAPOOR, 2001).

Esses alimentos considerados como funcionais apresentam um consumo cada vez mais em alta em razão de seus efeitos benéficos, seja para a prevenção de doenças ou para o atendimento das necessidades nutricionais básicas. Em razão do seu potencial nutritivo e do seu baixo custo, muitos frutos e plantas nativas do bioma cerrado têm sido utilizados como suplementação dietética pelo seu potencial nutricional e como tratamento alternativo ou complementar em diversas doenças, no entanto, nem todos tiveram seus efeitos sobre o organismo investigados. Portanto, justifica-se essa revisão integrativa com o objetivo de sumarizar os estudos realizados e descrever os resultados encontrados de forma clara e objetiva para servir como base para futuros ensaios clínicos dos efeitos da ingestão desses frutos pelo organismo humano.

Assim, este estudo objetivou identificar óleos, plantas e frutos pertencentes ao cerrado brasileiro com potencial nutricional para a alimentação humana por meio de estudos primários, de forma a responder a seguinte questão norteadora: Quais os óleos, plantas e frutos do bioma cerrado com potencial nutricional para serem utilizados na alimentação humana?

\section{Material e Métodos}

Trata-se de um estudo de revisão integrativa, que busca identificar na literatura científica resultados amplos, detalhados ou extensos que agrupa os achados de estudos desenvolvidos por meio de diferentes metodologias, permitindo aos revisores sintetizar resultados, considerar a filiação epistemológica de estudos empíricos incluídos e também a incorporação da aplicabilidade dos estudos encontrados na prática (SOUZA; SILVA; CARVALHO, 2010; SOARES, et al.,2014). Para a operacionalização desta pesquisa, foram realizadas as seguintes etapas: 1) Identificação da questão norteadora, 2) Seleção das fontes de pesquisa, 3) Seleção dos estudos relevantes ao considerar os critérios de inclusão e exclusão, 4) Extração dos dados por meio da síntese qualitativa, 5) Mapeamento dos resultados, com inclusão de tabelas e/ou gráficos na análise temática qualitativa, e 6) Divulgação dos resultados. A questão norteadora foi elaborada com base na estratégia $\mathrm{PVO}$, quando o "P" se refere a população, o "V" a variável de interesse e o "O" ao resultado, do inglês outcome. Dessa forma, ao considerar a população constituída pelos óleos, plantas e frutos, a variável de interesse relacionada ao bioma cerrado e o resultado como o potencial nutricional para a dieta humana, se chegou a seguinte questão norteadora para execução deste estudo: "Quais os óleos, plantas e frutos do bioma cerrado com potencial nutricional para utilização na dieta humana?".

A busca nas bases de dados ocorreu no período de janeiro a abril de 2019, realizada por dois pesquisadores independentes, mediante o uso do proxy licenciado pela Universidade Federal do Rio Grande do Norte, por meio da Coordenação de Aperfeiçoamento de Pessoal de Nível Superior (CAPES) (HTTP://www.capes.gov.br/), acessado via Portal de Periódicos CAPES (http://www-periodicoscapes-gov-br.ez18.periodicos.capes.gov.br/) nas seguintes bases de dados: Science Direct (Elsevier), Cumulative índex to nursing and Allied Health Literature (CINAHL) e na Biblioteca Virtual em Saúde (BVS).

Para a busca nas bases de dados foram utilizados os descritores indexados e controlados pelo Medical Subject Headings (MeSH) e não controlados com aplicação do operador booleano AND por meio de cruzamento único 1\# AND 2\# AND 3\#, a saber: 1\# ("Plants edible") AND 2\# ("Diet, Food and Nutrition") AND 3\# ("Cerrado").

Foram incluídos artigos completos, disponíveis nas bases de dados adotadas, que abordaram o uso de óleos, plantas e frutos do cerrado como fonte potencial nutricional, bem como estudos em todos os idiomas e sem delimitação temporal com o objetivo de abranger o máximo de publicações possíveis para compor os resultados. Excluiu-se do estudo artigos de revisão, teses, dissertações, cartas ao editor, resumos, opiniões de especialistas, resenhas, livros, capítulos de livros e monografias.

Para possibilitar a análise e extração dos resultados dos estudos selecionados, um instrumento específico foi elaborado para a coleta de dados que contem os seguintes itens: título do artigo, periódico, autores, país, idioma, ano de publicação, local do estudo, tipo de estudo, objetivos, amostra, resultados, grau de recomendação e nível de evidência.

Os estudos foram avaliados quanto ao nível de evidência e grau de recomendação por meio do índice de Oxford de March 2009, considerados apenas aqueles com grau de recomendação $\mathrm{B}$ ou superior e nível de evidência 2B ou superior, de forma a responder a questão norteadora do estudo.

O processo de busca e seleção dos artigos da amostra final encontra-se descrito na figura 1 . Os resultados foram analisados e sintetizados de modo descritivos e apresentados em formato de tabelas e figuras 
Figura 1. Fluxograma de seleção dos artigos para composição da amostra final da revisão.

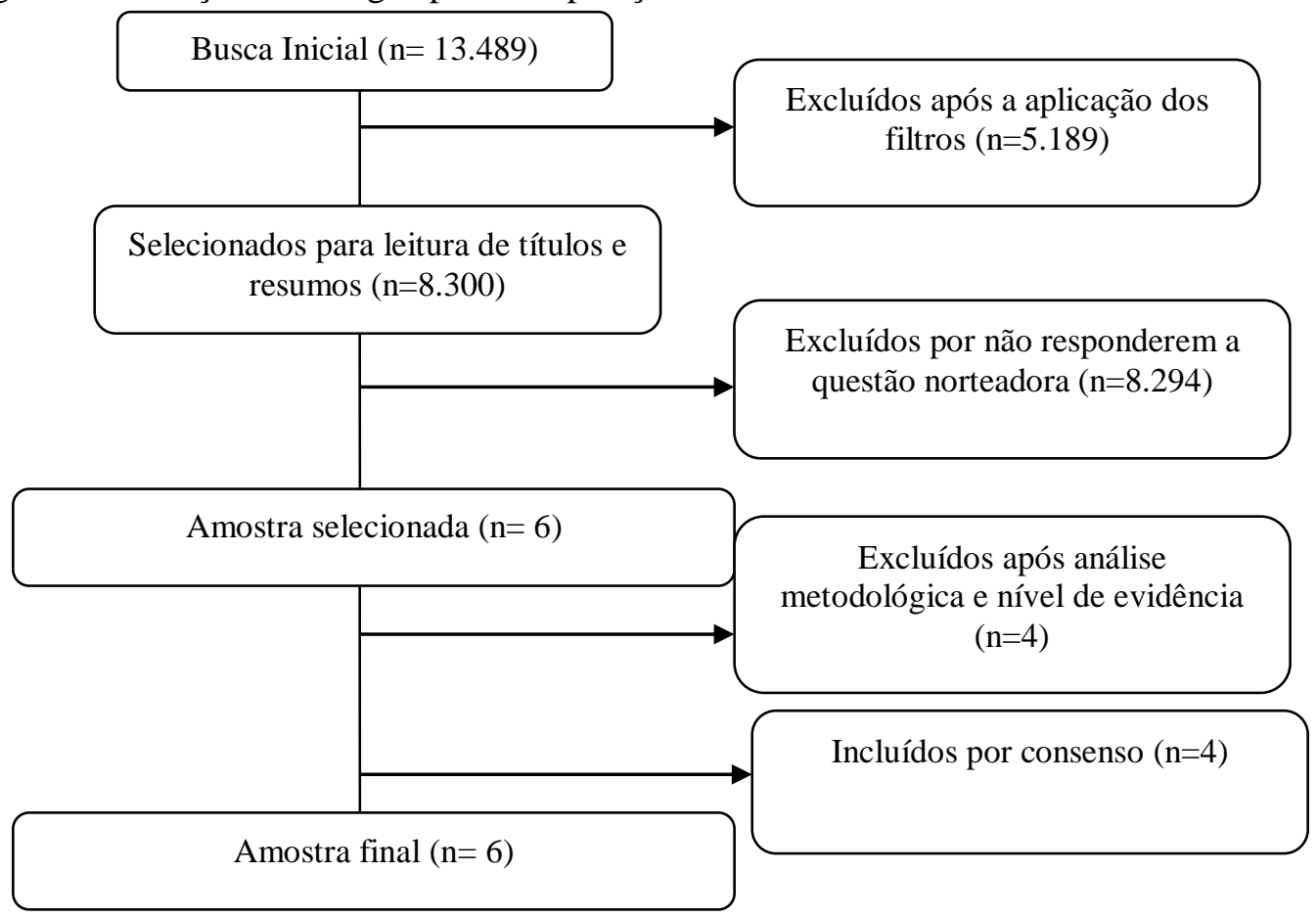

Fonte: Dados de pesquisa. Campo Grande/MS, Brasil, 2019.

\section{Resultados}

Dos 13.489 artigos iniciais, seis compuseram a amostra final para a análise dos resultados, todos publicados em inglês (100\%), com o Brasil como único local de execução de todos os estudos. Todos estavam disponíveis na base de dados CINAHL, publicados em um recorte temporal de 6 anos, de 2012 a 2018. Os periódicos que mais publicaram foram Journal of Ethnopharmacology $(28,5 \%)$ e Evidenc-Based Complementary $(28,5 \%)$.

A caracterização dos estudos selecionados para a composição da amostra final segue na tabela abaixo que foi elaborada de acordo com o instrumento elaborado especificamente para a coleta de dados (Quadro 1).

Os delineamentos de estudos mais utilizados foram os estudos experimentais com uso de modelos de animais $(83,3 \%)$, seguido pelo ensaio clínico randomizado controlado $(16,6 \%)$, com predomínio da abordagem quantitativa e todos com níveis de evidência 2B. Com relação aos graus de recomendação, todos apresentaram o grau $\mathrm{B}$, que refere uma recomendação consistente de estudos nível 2 ou 3 (MARCH, 2009), conforme tabela 1.

Tabela 1. Categorização da amostra conforme delineamento de pesquisa, abordagem do estudo, grau de recomendação e nível de evidência de acordo com o Índice de Oxford 2009. Campo Grande/MS, 2019 (n=6).

$\begin{array}{rr}\text { Variáveis } & \mathbf{n}(\mathbf{\%}) \\ \text { Delineamento de pesquisa } \\ \text { Estudos experimentais } \\ \text { Ensaio Clínico Randomizado, Controlado } \\ \text { Abordagem do estudo } \\ \text { Quantitativo } \\ \text { Grau de Recomendação } \\ \mathrm{B} \\ \text { Nível de Evidência } \\ 2 \mathrm{~B} & 6(105,7) \\ & 6(10,6) \\ \end{array}$

Fonte: Dados de pesquisa. Campo Grande/MS, Brasil, 2019.

A caracterização dos estudos selecionados para a composição da amostra final segue na tabela abaixo que foi elaborada de acordo com o instrumento elaborado especificamente para a coleta de dados (Quadro 1). 
Quadro 1. Caracterização dos estudos selecionados para compor a amostra final. Campo Grande/MS, 2019 ( $n=6$ ).

\begin{tabular}{|c|c|c|c|c|c|}
\hline Estudo & $\begin{array}{l}\text { Referênci } \\
\text { a/País/An } \\
\text { o }\end{array}$ & Periódico & Objetivo & $\begin{array}{c}\text { Método/ } \\
\text { Nível de } \\
\text { Evidência } \\
\end{array}$ & Principais Resultados \\
\hline $\mathrm{A}$ & $\begin{array}{c}\text { Orsi et.al } \\
\text { Brasil } \\
(2012)\end{array}$ & $\begin{array}{c}\text { Journal of } \\
\text { Ethnopharmacology }\end{array}$ & $\begin{array}{l}\text { Investigar os efeitos } \\
\text { antidirreicos e anti- } \\
\text { ulcerosos do extrato } \\
\text { etanólico do caule e } \\
\text { da polpa de } \mathrm{H} \text {. } \\
\text { stignocarpa }\end{array}$ & $\begin{array}{c}\text { Estudo } \\
\text { Experimental em } \\
\text { modelo animal } \\
\text { B/2B } \\
\text { JADAD: } \\
\text { 3pontos }\end{array}$ & $\begin{array}{l}\text { O tratamento com polpa da } \\
\text { fruta apresentou efeito } \\
\text { antidiarréico, protetor gástrico } \\
\text { e duodenal sem qualquer sinal } \\
\text { de toxicidade. }\end{array}$ \\
\hline B & $\begin{array}{l}\text { Bento et } \\
\text { al } \\
\text { Brasil } \\
(2014)\end{array}$ & $\begin{array}{c}\text { Nutrition, Metabolis } \\
\text { me Cardiovascular } \\
\text { Diseases }\end{array}$ & $\begin{array}{l}\text { Avaliar o efeito no } \\
\text { perfil lipídico e } \\
\text { oxidativo em } \\
\text { invidíduos com dieta } \\
\text { suplementada com } \\
\text { amêndoas de Baru. }\end{array}$ & $\begin{array}{l}\text { Estudo } \\
\text { experimental, } \\
\text { com invidíduos } \\
\text { entre 21-57 anos } \\
\text { B/2B } \\
\text { JADAD: } \\
\text { 3pontos }\end{array}$ & $\begin{array}{l}\text { O óleo de baru demonstrou } \\
\text { efeito hipolipêmico com } \\
\text { redução de LDL. }\end{array}$ \\
\hline $\mathrm{C}$ & $\begin{array}{l}\text { Veloso et } \\
\text { al } \\
\text { Brasil } \\
(2014)\end{array}$ & $\begin{array}{c}\text { Journal of } \\
\text { Ethnopharmacology }\end{array}$ & $\begin{array}{l}\text { Investigar os efeitos } \\
\text { da planta no } \\
\text { tratamento de } \\
\text { doenças anti- } \\
\text { inflamatórias e } \\
\text { dinsfunção } \\
\text { metabólica. }\end{array}$ & $\begin{array}{c}\text { Estudo } \\
\text { Experimental em } \\
\text { modelo animal } \\
\text { B/2B } \\
\text { JADAD: } 3 \\
\text { pontos }\end{array}$ & $\begin{array}{l}\text { O extrato da planta demonstrou } \\
\text { redução de adipócitos e } \\
\text { aumentou a tolerância a } \\
\text { glicose. Auxiliou também na } \\
\text { redução de mediadores } \\
\text { inflamatórios no tecido } \\
\text { muscular, adiposo e hepático. }\end{array}$ \\
\hline $\mathrm{D}$ & $\begin{array}{c}\text { Romero et } \\
\text { al } \\
\text { Brasil } \\
(2015)\end{array}$ & $\begin{array}{c}\text { Nutrición } \\
\text { Hospitalaria }\end{array}$ & $\begin{array}{l}\text { Avaliar a composição } \\
\text { do Pequi e atividade } \\
\text { antioxidante in vivo e } \\
\text { in vitro de sua polpa. }\end{array}$ & $\begin{array}{c}\text { Ensaio } \\
\text { Experimental em } \\
\text { modelo animal } \\
\text { B/2B } \\
\text { JADAD: } 3 \\
\text { pontos }\end{array}$ & $\begin{array}{l}\text { Dietas enriquecidas com o } \\
\text { buriti apresentaram maior teor } \\
\text { antioxidante quando } \\
\text { comparadas com dieta padrão, } \\
\text { podendo ser cliassificado como } \\
\text { um alimento funcional com } \\
\text { potencial antioxidante. }\end{array}$ \\
\hline $\mathrm{E}$ & $\begin{array}{l}\text { Nunes } \\
\text { et.al } \\
\text { Brasil } \\
(2018)\end{array}$ & $\begin{array}{l}\text { Plant Foods for } \\
\text { Human Nutrition }\end{array}$ & $\begin{array}{l}\text { Avaliar o efeito da } \\
\text { semente de acromia } \\
\text { aculeata sobre a } \\
\text { glicemia na Diabetes } \\
\text { tipo } 2 \text { e o tecido } \\
\text { adiposo em modelo } \\
\text { animal. }\end{array}$ & $\begin{array}{c}\text { Ensaio } \\
\text { Experimental em } \\
\text { modelo animal } \\
\text { B/2B } \\
\text { JADAD: } 3 \\
\text { pontos }\end{array}$ & $\begin{array}{l}\text { A semente demontrou efeitos } \\
\text { hipoglicemiantes. }\end{array}$ \\
\hline $\mathrm{F}$ & $\begin{array}{l}\text { Reis et. al } \\
\text { Brasil } \\
(2018)\end{array}$ & $\begin{array}{c}\text { Evidenc-Based } \\
\text { Complementary na } \\
\text { Alternative Medicine }\end{array}$ & $\begin{array}{l}\text { Avaliar o perfil de } \\
\text { ácidos graxos } \\
\text { presentes no óleo de } \\
\text { Baru e investigar seus } \\
\text { efeitos na função } \\
\text { hepática e nos } \\
\text { marcadores séricos de } \\
\text { risco cardiovascular } \\
\text { através de modelo } \\
\text { animal. }\end{array}$ & $\begin{array}{l}\text { Ensaio } \\
\text { experimental } \\
\text { em modelo } \\
\text { animal } \\
\text { B/2B } \\
\text { JADAD: } 3 \\
\text { pontos }\end{array}$ & $\begin{array}{l}\text { O óleo de Baru demonstrou } \\
\text { efeitos na redução de danos no } \\
\text { fígado, e possui potencial para } \\
\text { ser considerado um alimento } \\
\text { funcional. }\end{array}$ \\
\hline
\end{tabular}

Fonte: Dados de pesquisa. Campo Grande/MS, Brasil, 2019.

Os estudos analisados foram organizados de acordo com as categorias identificadas nos resultados da amostra final: estudos com frutos, estudos com óleo e estudos com plantas (Tabela 2). 
Tabela 2. Organização dos estudos de acordo com o produto/subproduto analisado. Campo Grande MS, 2019 (n= 6).

\section{Estudo}

Orsi et.al, 2012

Bento et al, 2014

Veloso et al., 2013

Romero et al, 2015

Nunes et.al. 2018

\section{Produto/subproduto analisado}

\author{
Frutos e plantas
}

Frutos

Plantas

Frutos

Óleos

Óleos

Fonte: Dados de pesquisa. Campo Grande/MS, Brasil, 2019.

Estudos com os seguintes frutos e plantas foram encontrados: Jatobá-do-cerrado (Hymenaea stigonocarpa Mart. ex Hayne); Buriti (Mauritia flexuosa L.F); Baru (Dipteryxalata Vog.); Macaúba (Acromia Aculeata); e Cipó-de-são-joão (Pyrostegia venusta (Ker Gawl) Miers). Apesar de haver na literatura uma gama de estudos sobre frutos do cerrado, grande parte deles se limitam apenas à caracterização físicoquímica, e não a realização de estudos experimentais ou ensaios clínicos com o objetivo de avaliar o efeito da ingestão desses frutos no organismo, o que justifica a pequena quantidade de artigos encontrados.

\section{Discussão}

\subsection{Estudos com frutos}

Foram utilizadas as espécies Hymenaea stigonocarpa Mart. ex Hayne; Mauritia flexuosa L.F $e$ Dipteryxalata Vog. para análises com frutos, conhecidas popularmente como Jatobá-do-Cerrado, Buriti e Baru, respectivamente.

Orsi et al investigaram os efeitos antidiarreicos e antiulcerosos a partir da dieta preparada com a farinha da polpa dos frutos do Baru em modelos experimentais de roedores. Lesões gástricas e duodenais foram induzidas em ratos Wistar albinos machos $(\mathrm{n}=8)$ que permaneceram em jejum por 18 horas apenas com livre acesso à água. Foi realizada uma incisão cirúrgica no abdômen de cada camundongo sob anestesia. O estômago e o duodeno foram inoculados com ácido acético e depois banhados com solução salina para remoção do mesmo. $\mathrm{O}$ abdômen foi fechado e os ratos foram alimentados normalmente com a farinha da polpa da farinha do Baru por um período de 7 ou 14 dias consecutivos. A farinha foi preparada por meio de procedimentos de desidratação, pulverização e misturada com a alimentação padrão. A atividade de toxicidade subaguda também foi avaliada após o experimento por diversos parâmetros analisados durante os 14 dias de tratamento.

Como resultado, a dieta com farinha da polpa do fruto não apresentou efeito significativo na redução do tamanho da lesão gástrica em relação ao controle positivo
(Solução salina) e negativo (Lansoprazol) durante os 7 primeiros dias de tratamento. No entanto, no final dos 14 dias a dieta auxiliou na redução de $61 \%$ da lesão gástrica produzida em relação ao controle negativo (Solução salina) e controle positivo (Lansoprazol) que reduziu em $81 \%$ a área da lesão. Em relação a lesão duodenal, a dieta com a farinha da polpa reduziu de maneira significativa a lesão comparado ao controle negativo e positivo somente nos primeiros 7 dias com $71 \%$ de redução.

Além disso, a dieta com a farinha da polpa também foi capaz de manter o nível da enzima antioxidante glutationa que está intimamente ligada a prevenção de úlceras duodenais, no período de 7 e 14 dias. Tais resultados reforçam o acentuado efeito da dieta com a polpa de Baru na inibição dessas lesões, com promoção de cura de doenças gástricas e duodenais em ratos (ORSI, et al, 2012).

$\mathrm{Na}$ análise de toxicidade, a dieta com a farinha da polpa não produziu nenhuma mudança de comportamento durante o tratamento. $\mathrm{O}$ peso corporal progrediu de maneira semelhante em todos os grupos e as análises bioquímicas para as funções hepáticas e renais não revelaram alteração (ORSI, et.al., 2012).

Os resultados apresentados podem ser justificados em razão da natureza polifenólica da Hymenia Stignocarpa, que é composta principalmente por flavonóides e taninos condensados. Atividades anti-ulcerosas promovida pelo papel dos flavonóides já foram elencadas em estudos anteriores com a proteção da mucosa gástrica da ação de diversos agentes ulcerogênicos. Sua ação ocorre a partir da inibição de enzimas específicas relacionadas ao processo inflamatório e da inibição da produção de óxido nítrico. Além disso, a ação antioxidante e citoprotetora dos taninos também auxiliam no efeito redutor das lesões gastrointestinais (MOREIRA et al., 2004; OKUDA, 2005, WACH et al., 2007).

O Jatobá do Cerrado apresentou em alguns estudos ser uma fonte significativa de fibra bruta, com quantidades consideráveis também de potássio, magnésio, zinco e cálcio, que podem ter contribuído para a as ações citoprotetoras, uma vez que nutrientes como vitaminas antioxidantes, minerais, fitoquímicos, fibras e entre outros, participam 
diretamente do efeito protetor gastrointestinal que algumas frutas possuem (BATISTA, 2011; MARIN et al., 2009). Seu fruto tem sido consumido tanto na forma in natura como também na forma de geléia, licor, farinha para bolos, pães e minguaus e sua farinha também tem sido utilizada para a preparação de biscoitos, como substituição de parte da farinha convencional, tendo como resultados biscoitos isentos de açucar e fonte rica de fibra alimentar (SILVA et al., 2001a; SILVA et al., 2001b).

Romero et al. pesquisaram a atividade antioxidante do Buriti bem como sua composição química. A dieta padrão dos roedores foi suplementada a partir da trituração da polpa do fruto na proporção de $10 \mathrm{~g}$ polpa/100g da ração. Ratos de ambos os sexos com aproximadamente $36 \mathrm{~g}$ foram distribuídos de forma aleatória em 4 grupos e tratados por 60 dias com a dieta padrão ou a enriquecida com a polpa do Buriti. Após 60 dias os animais foram sacrificados, amostras de sangue foram coletadas para avaliação da peroxidação lipídica no plasma sanguíneo, o fígado foi retirado para fins de análise de oxidação e os rins para avaliação de peroxidação lipídica (ROMERO et al., 2015).

A análise da composição química do fruto demonstrou um alto teor calórico $(592 \mathrm{kcal} / 100 \mathrm{~g}$ na polpa e $363 \mathrm{kcal} / 100 \mathrm{~g}$ na alimentação enriquecida) e altas concentrações de lipídios $(45,7 \mathrm{~g} / 100 \mathrm{~g}$ na polpa e $5,9 \mathrm{~g} / 100 \mathrm{~g}$ na alimentação enriquecida), carboidratos $(36,3 \mathrm{~g} / 100 \mathrm{~g}$ na polpa e $57,7 \mathrm{~g} / 100 \mathrm{~g}$ na alimentação enriquecida), proteínas $(9,0 \mathrm{~g} / 100 \mathrm{~g}$ na polpa e $20,0 / 100 \mathrm{~g}$ na alimentação enriquecida) e carotenóides $(23,9 \mathrm{~g} / 100 \mathrm{~g}$ na polpa e $5,3 \mathrm{~g} / 100 \mathrm{~g}$ na dieta enriquecida). Tanto na polpa do Buriti quanto na deita enriquecida foram encontrados quantidades expressivas de compostos fenólicos semelhante a outros estudos realizados, porém, suas concentrações são bastante variáveis por conta da influência de fatores como maturação, espécie, origem geográfica, estágio de crescimento, condições de coleta e armazenamento dos frutos (TOMÁS; BARBERÁN; ESPIN, 2001).

A alta concentração de lipídios presente no Buriti, principalmente do ácido oleico, identificado durante o estudo, é desejada em termos de nutrição e culinária, pois auxilia na redução dos níveis de colesterol e apresenta alta establididade durante seu aquecimento, evitando aalteração da sua composição (YUYAMA, 1998; LUZIA, 2012). Além disso, a quantidade elevada de carotenóides presentes na polpa confere ao Buriti caráter funcional à medida que esse componente exerce um papel fundamental na visão, crescimento, desenvolvimento ósseo e manutenção do tecido epitelial e sua ingestão a partir de geleias tem sido indicada para auxiliar no tratamento de hipovitaminose A em razão de atuar como precussor dessa vitamina (ALMEIDA, 1995; EMBRAPA, 2006; OLIVEIRA, 2011).

Alguns estudos realizados apresentam variação quanto a composição nutricional da polpa do Buriti que pode ser justificada pela diferença entre as várias regiões do país e condições de colheita, aquisição, secagem e armazenamento da fruta (YUYAMA, 1998; LUZIA, 2012; ROMERO et al., 2015).

$\mathrm{Na}$ análise de antioxidação o estudo demonstrou a partir de marcadores químicos que a atividade antioxidante foi mais estimulada no grupo alimentado com a dieta com polpa do fruto. Esse papel antioxidante pode estar relacionado com a quantidade expressiva de vitamina $\mathrm{E}$ presente no Buriti, considerada uma fonte exógena de propriedades antioxidantes. Apesar desse efeito não ser exercido de forma isolada em razão da ação do sistema natural de defesa antioxidante com as enzimas superóxido dismutase, catalase, glutationa peroxidase, a dieta com a polpa do Buriti pode auxiliar quando consumido de forma suplementar (LUZIA, 2012; ROMERO, et al. 2015).

Alguns autores já mencionam a utilização do fruto na preparação de doces e outros subprodutos artesanais. Além disso, diferentes tipos de sorvetes, cremes, geléias, licores e vitaminas de sabores exóticos podem ser produzidos com sua polpa. Assim, o uso do Buriti em dietas nutricionalmente equilibradas pode ser apoiado com objetivo de exercer efeitos preventivos e benéficos ao organismo humano, mantendo os níveis de espécies reativas de oxigênio e nitrogênio adequados (ROMERO, et al. 2015; SILVA, 2011).

Não houve alterações estatisticamente significantivas no marcador químico resultante de oxidações lipídicas Malonaldeído (MDA, do plasma, rins ou fígado dos ratos devido ao consumo de alimentos enriquecidos com polpa de Buriti, portanto, não houve aumento de processos oxidativos prejudiciais ao organismo (ROMERO et al., 2015).

O Buriti pode ser classificado como um alimento funcional em razão da presença de substância bioativas com teor antioxidante, proteicas e lipídicas, podendo ser utilizada para a prevenção de diversas doenças por meio da suplementação dietética com a polpa do fruto (ROMERO, et al. 2015).

Bento et al avaliaram os efeitos da amêndoa do Baru na redução de lipídios séricos, bem como seu potencal de oxidação em pessoas levemente hipercolesterolêmicas. Um total de 25 indivíduos com idade entre 21 e 57 anos foram recrutados por meio da distribuição de folhetos. Foram excluídos do estudo pessoas tabagistas, com histórico de doenças crônicas, que participavam em programas de perda de peso, uso de medicamentos hipolipemiantes ou outros medicamentos conhecidos capazes de alterar as funções lipídicas, com histórico de consumo frequente de nozes, aversão ou alergia a mesma.

Os indivíduos foram designados de forma aleatória para receber um dos dois tratamentos em períodos alternados por 6 semanas: uma suplementação com as amêndoas de Baru 20g/dia e outra suplementação placebo com uma cápsula de amido de milho/dia. As análises foram realizadas com base nas amostras de sangue coletadas em jejum de 12 horas no início do estudo e no final da cada tratamento.

Dos 25 indivíduos que atenderam aos critérios de elegibilidade, apenas 20 foram incluídos na análise, 8 homens e 12 mulheres. Os participantes mantiveram a ingestão de energia, macronutrientes e fibras alimentares ao longo do estudo. A suplementação dietética com as amêndoas de Baru resultou em uma tendência de aumento da vitamina $\mathrm{E}$ e ácidos graxos poliinsaturados. Comparado ao placebo, a ingestão das amêndoas de Baru reduziu significativamente as concentrações séricas de Colesterol Total, a partir da redução da concentração sérica de LDL $(9,4 \%)$. O consumo de $20 \mathrm{mg}$ de amêndoas de Baru não foi o 
suficiente para produzir mudanças ou ocasionar processos de de proteína e 47,9\% de carboidratos) e 160 g de AKO; Grupo oxidação (BENTO, et al., 2014).

$\mathrm{V}(\mathrm{NAH})$ : ratos não diabéticos que receberam da dieta 3 (

Apesar do alto valor calórico da amêndoa de Baru 37,4\% de lipídios, 14,7\% de proteína e 47,9\% de carboidratos) (110 kcal/dia), não houve alterações no peso corporal dos e $160 \mathrm{~g}$ de AKO. A glicemia de jejum foi monitorada nos dias indivíduos, provavelmente em função do alto conteúdo de $0,7,14,21$ e 28 . No final do tratamento os ratos foram fibras e proteínas da amêndoa aumentar a saciedade e reduzir submetidos a um jejum de 12 horas e anestesiados para coleta o consumo de outros alimentos com a manutenção da energia de sangue.

habitual (BENTO et al., 2014). A redução da concentração de LDL promovida pelo consumo de amêndoas representa uma diminuição de $9,4 \%$ no risco de Doenças Cardiovasculares (BENTO et al., 2014). Outros estudos realizados com a amêndoa de Baru e macadâmias apresentaram um nível maior de redução de LDL, porém, as porções oferecidas em cada intervenção foram muito maiores em comparação com a testada no presente estudo (BENTO et al., 2014).

Dados do estudo sugerem que a redução do colesterol total e da concentração sérica de LDL pode ser explicada pela sinergia entre o aumento de ácidos graxos insaturados, fibras alimentares e compostos bioativos presentes nas amêndoas de Baru. $\mathrm{O}$ estudo conclui que a ingestão de $20 \mathrm{~g}$ das amêndoas na dieta habitual de indivíduos levemente hipercolesterolêmicos possui um efeito benéfico sobre os níveis séricos de lipídios e pode contribuir para a redução do risco de doenças cardiovasculares, sugerindo a integração das amêndoas ao manual de oleaginosas recomendadas na dieta para redução dos riscos de DCV (BENTO et al., 2014).

A semente ou amêndoa do Baru possui gosto semelhante ao do amendoin, e além das propriedades terapêuticas já evidenciadas, também lhe é atribuído propridades afrodisíacas pela população da região. Pode ser consumida crua ou na forma de torrada, e substitui com a mesma equivalência a castanha-de-caju, servindo como ingrediente em algumas receitas de pé-de-moleque, rapadura e paçoquinha. Embora possua considerável potencial econômico, o fruto não possui sua comercialização estabelecida na cidade, e é melhor apreciado como planta nativa nas fazendas do centro-oeste. Portanto, seu consumo pela população deve ser mais estimulado (AVIDOS; FERREIRA, 2002; RODRIGUES, 2004).

\subsection{Estudos com óleo}

Foram utilizadas as espécies Dipteryxalata Vog. e Acromia Aculeata para análises com óleos, conhecidas popularmente como Baru e Palmeira Macaúba, respectivamente.

Nunes et al avaliaram os efeitos do óleo da semente de Macaúba sobre a glicemia e o tecido adiposo em ratos com Diabetes Mellitus tipo 2. A indução do Diabetes Mellitus foi feita a partir da inoculação de estreptozotocina (STZ) via intraperitonial em parte dos ratos. Os animais foram divididos aleatoriamente em cinco grupos $(n=8)$ da seguinte forma: Grupo I recebeu dieta padrão; Grupo II (DBC): controle diabético, também recebeu dieta padrão; Grupo III (DAL): ratos diabéticos que receberam da dieta 2 $(9,4 \%$ de lipídios, $14,7 \%$ de proteína e $75,9 \%$ de carboidratos) e 40g de AKO; Grupo IV(DAH): ratos diabéticos que receberam a dieta 3 (37,4\% de lipídios, $14,7 \%$

O resultado do estudo indicou que a introdução do óleo da semente de Macaúba como substituto parcial do carboidrato na dieta dos camundongos teve um efeito relevante na redução da glicemia. Após a primeira semana da dieta, tanto na baixa como na alta dose ( $40 \mathrm{~g}$ e $160 \mathrm{~g}$ ), o nível de glicose nos ratos diabéticos começou a diminuir significativamente. Os grupos que receberam da dieta com o óleo após os 28 dias diminuíram em aproximadamente 50\% a glicemia em comparação com o grupo de ratos diabéticos que receberam da dieta padrão. Os dados sugerem um efeito benéfico do óleo da semente de Macabúba sobre os níveis glicêmicos nos indivíduos com Diabetes Mellitus tipo 2, e novos estudos são necessários com maior período de análise para avaliar a possibilidade de alcançar níveis glicêmicos normais com a administração contínua do mesmo.

$\mathrm{O}$ peso corporal dos grupos que receberam a dieta com o óleo também foram significativamente menores, que pode ser explicado devido ao maior catabolismo de gorduras e proteínas em resposta à resistência insulínica no tecido muscular e adiposo (SUNDARAM et al., 2013).

Em relação ao perfil de ácidos graxos, o ácido láurico foi o óleo mais abundante encontrado na semente de Macaúba, semelhante a outros estudos já realizados, e este é considerado como componente benéfico para a saúde em razão dos seus efeitos antiinflamatórios e auxílio no controle de peso. Não houve acúmulo de ácidos graxos de cadeia média no tecido adiposo dos ratos alimentados com a ingestão do óleo, o que contribui para maior economia do metabolismo dos carboidratos e evita o aumento da adiposidade hepática. (NUNES, et al., 2018; AMARAL, 2007)

Quantidades expressivas de ácidos graxos foram encontradas em outros estudos realizados, sugerindo que a amêndoa de Macaúba seja utilizada na preparação de alimentos funcionais, podendo ser consumida de forma in natura ou na forma de doces. Além disso, também é considerada uma fonte promissora de energia para substituir parcialmente os carboidratos em dietas com a manutenção dos níveis de glicose no sangue em indivíduos com Diabetes Tipo 2 (COIMBRA, 2010; NUNES et al., 2018).

Reis et al. analisaram o perfil de ácidos graxo presentes no óleo das amêndoas de Baru e investigaram seus efeitos na função hepática, nos marcadores séricos de risco cardiovascular e peroxidação lipídica em camundongos hiperlipidêmicos.

Ratos Wistar machos $(n=40)$ pesando em média $25 \mathrm{~g}$ foram divididos aleatoriamente em quatro grupos da seguinte forma: o grupo controle recebeu água potável; um grupo recebeu apenas o óleo de Baru; um grupo recebeu emulsão lipídica;e o outro grupo recebeu emulsão lipídica e o óleo da amêndoa de Baru, administrado por gavagem na dose de $1 \mathrm{~g} / \mathrm{kg} / \mathrm{dia}$ e $10 \mathrm{~mL} / \mathrm{kg}$, respectivamente por um período de 15 semanas. Ao final do tratamento os animais 
foram submetidos ao jejum de 12 horas e depois anestesiados para a coleta de sangue da aorta abdominal e após isso sacrificados para a coleta do fígado e da aorta.

O resultado do perfil lipídico do Baru apresentou predomínio de ácidos graxos monoinsaturados seguido por ácidos graxos poliinsaturados . Entre os insaturados, o ácido oleico foi o mais abundante, enquanto entre os poliinsaturados, o ácido linoléico foi o que predominou. Os ácidos graxos saturados foram observados em menor quantidade como o ácido palmítico, seguido do lenhoreico, esteárico, bênico, e ácidos araquídicos. O conteúdo encontrado no estudo foi inferior a outras pesquisas, no entanto, essa diferença é esperada, pois fatores como variedade genética, clima, época do ano, tipo de solo, armazenamento, processo de extração e análise podem afetar diretamente a composição química do óleo de várias espécies de plantas (SILVA, 2011). Devido ao perfil lipídico apresentado e a predominância de lipídios insaturados, o óleo de Baru demonstrou-se adequado para o consumo humano e pode ser indicado para o uso na culinária doméstica (TOGASHI; SCARBIERI, 1993).

Houve uma diminuição da peroxidação lipídica na aorta abdominal no grupo que recebeu o óleo de Baru em comparação com o grupo controle, devido à processos antioxidantes no sangue da aorta, sugerindo que o óleo de Baru pode ser um alimento com potencial de aplicação em condições associadas ao estresse oxidativo vascular (RAJEDRAN; NANDAKUMAR; RENGARAJAN et al., 2014; REIS, et al., 2018).

A administração de emulsão lipídica induziu a uma drástica remodelação morfológica nos tecidos hepáticos dos camundongos que foram atenuados pelo tratamento com óleo de baru. Dessa forma, o óleo de Baru apresentou repercussões positivas na estrutura hepática, especialmente na atuação de hepatócitos degenerativos. Embora muito pouco compreendidos, tais efeitos benéficos podem estar relacionados à possível ação dos ácidos graxos insaturados e compostos antioxidantes importantes na modulação das atividades metabólicas hepáticas envolvidas no processo de lipotoxicidade (HANKE et al., 2013).

$\mathrm{O}$ estudo conclui que o óleo de Baru possui uma boa composição de ácidos graxos com alto valor. Os ácidos graxos monoinsaturados foram predominantes, enquanto ácidos graxos pollinsaturados e saturados foram detectados em níveis menores. Ao diminuir a peroxidação lipídica vascular e danos morfológicos hepáticos em camundongos dislipidêmicos, o óleo de Baru também pode ser sugerido como um alimento funcional (REIS et al., 2018)

\subsection{Estudos com plantas}

Apenas um estudo com plantas foi encontrado para a análise dietética. A espécie utilizada foi a Pyrostegia venusta (Ker Gawl) Miers, conhecida popularmente como flor-desão-joão ou cipó-de-são-joão.

Veloso et al investigaram o efeito da suplementação dietética com extrato hidroetanólico das flores de Pyrostegia venusta sobre disfunções inflamatórias e metabólicas induzidas por uma dieta rica em carboidratos refinados.

Os extratos hidroetanólicos da planta foram preparados a partir de processos de secagem, pulverizaçção e maceração com solução hidroalcoólica. Camundongos machos entre 5 a 7 semanas foram divididos inicialmente em dois grupos: (I) grupo controle (animais alimentados com ração padrão); (II) grupo da dieta hiper calórica (animais alimentados com alta quantidade de carboidratos refinados). Após 8 semanas de intervenção dietética os camundongos foram submetidos ao teste oral de intolerância a glicose (TOTG). Na nona semana, o grupo da dieta hiper calórica foi dividido aleatoriamente, parte dos animais continuou a receber a dieta padrão e a outra passou a ser alimentada com a dieta padrão e suplementação com $300 \mathrm{mg} / \mathrm{kg} / \mathrm{dia}$ do extrato da cipó-de-são-joão, grupo (PvHE). Após isso, todos os grupos fizeram novamente o TOTG e depois foram sacrificados.

Os resultados indicaram que nenhuma das dietas apresentaram diferenças quanto ao ganho de peso nos animais. No entanto, a adiposidade aumentou $67 \%$ nos ratos alimentados com a dieta hipercalórica em relação ao controle enquanto os ratos alimentados com a dieta suplementada com a planta apresentou uma redução de $28,4 \%$ na adiposidade quando comparados com este grupo. A dieta com PvHE apresentou uma área e características das células gordurosas semelhantes aos animais alimentados com a dieta controle (VELOSO et al., 2014).

É possível atribuir esses efeitos de redução na adiposidade a presença de taninos e flavonoides nas flores de cipó-de-são-joão, que de acordo com alguns estudos são responsáveis por inibir a expressão de lipoproteínas e atividades pró-aterogênicas (ALLISTER et al., 2005).

Os animais alimentados com a dieta PvHE apresentaram um aumento da sensibilidade à insulina e melhora na intolerância à glicose em comparação com os outros grupos. É provável que esses achados estejam relacionados à diminuição de um estado pró-inflamatório controlados pela redução das adipocinas pró-inflamatórias (OLEFSKY; GLASS, 2010).

A suplementação dietética com flores de cipó-desão-joão também teve um efeito significativo com relação as citocinas responsáveis indução de respostas inflamatórias no tecido adiposo e no fígado quando comparadas com o grupo apenas com a dieta hiper calórica. Enquanto nesse grupo houve aumento nos níveis de TNF- $\alpha$, IL-6, IL-10, IL-4 e IL13, no grupo PvHE houve uma diminuição, com exceção da IL-10, considerada uma adipocina antiinflamatória que permaneceu com a sua concentração. Um dos motivos para tal achado se dá pela atuação de uma substância presente na planta chamada acacetina, que inibe o processo de transcrição das adipocinas inflamatórias.

Dessa forma, pode-se concluir que a dieta a base do extrato metanólico das flores de Pyrostegia venusta melhora a tolerância à glicose está associada a uma modulação negativa do processo inflamatório a nível local e sistêmico, com efeitos benéficos no tratamento da disfunção inflamatória e metabólica induzida por dieta rica em carboidratos refinados. Sugere-se que inibição de vias próinflamatórias que aumentam a resistência à insulina seja um possível alvo para intervenção terapêutica (VELOSO, et al., 2014).

Os frutos nativos ocupam um lugar de destaque no 
bioma do cerrado, que possui mais de 12 mil espécies catalogadas com destaque para as espécies frutíferas e plantas medicinais. Apesar de mais de 58 serem conhecidas e utilizadas pela população local, menos da metade foram estudadas, difundidas e são consumidas de forma regular pela população no geral, devido o desconhecimento sobre seu potencial nutricional e efeito terapêutico a partir da suplementação dietética seja através da polpa ou amêndoa do fruto.

As espécies nativas do cerrado são utilizadas com fins madeireiros, tintorial, medicinal, ornamental entre outros. Das espécies analisadas, o Pequi, o Baru, o Buriti e a Macaúba são as mais reconhecidas pela população com relação ao uso alimentar. Apesar de resultados com enfoque terapêutico apresentados pelos estudos encontrados, os frutos nativos também são capazes de superar as fontes tradicionais de vitaminas e minerais de frutos como a laranja-pera, o limão verde, abacate, banana e a goiaba (ALMEIDA, 1995; OLIVEIRA, 2011).

Estudos científicos com relação ao potencial alimentício de espécies nativas são necessários à medida que agregam valor econômico aos mercados e também pode ser uma alternativa viável para a sustentabilidade de pequenos e médios agricultores, auxiliando no aumento de renda familiar (EMBRAPA, 1994; OLIVEIRA, 2011). Alguns estudos sobre a aceitabilidade de receitas a partir dos frutos do cerrado evidenciaram que já existe por parte da população boa aceitabilidade e até mesmo intenção de compra de algumas preparações como sorvetes, coockies, geléias, pães e bolos (RODRIGUES, 2004).

A atividade a partir da utilização de plantas nativas do cerrado tem sido predominantemente extrativista, seja do uso dos frutos para a alimentação, atividade madeireira ou artesanato, diminuindo ainda mais a oferta desses vegetais. Assim, há uma necessidade de adoção de alternativas que viabilizem o uso sustentado desse bioma através da preservação de sua área e cultivos experimentais visando a produção desses frutos em larga escala e posteriormente sua comercialização a partir de melhoramentos genéticos que atendam as demandas exigidas pelo mercado brasileiro com vistas a garantir o conhecimento sobre o potencial nutricional desses frutos por parte das populações das outras regiões do país e seu melhor aproveitamento na culinária (SILVA, 2011).

Uma das limitações do estudo foi a pequena quantidade de artigos encontrados, pois ainda são poucos os estudos com caráter experimental que investigam o uso de frutos do bioma cerrado na alimentação, incluído ensaios de citoxicidade. Dessa forma, sugere-se que mais estudos relacionados a esse tema sejam realizados a fim de contribuir para a exploração sustentada desses frutos nos estados onde esse bioma ocorre.

$\mathrm{O}$ presente estudo permitiu identificar ações antiinflamatórias, antihiperlipêmicas, antidiarreicas, antiulceroas e antihiperglicêmicas a partir da ingestão do fruto, do extrato da flor ou do óleo de algumas espécies do cerrado.

O jatobá do cerrado, o buriti, o baru, a palmeira de macaúba e a cipó-de-são-joão possuem valores nutricionais e curativos. O cerrado possui óleos, plantas e frutos que exercem efeitos benéficos ao organismo quando consumidos como suplementação dietética. Esses efeitos permitem que os frutos sejam caracterizados como alimentos funcionais e sua ingestão deve ser estimulada, a fim de prevenir doenças e também atender as necessidades funcionais básicas do organismo.

Existem muitas espécies pertencentes ao bioma cerrado que auxiliam na viabilidade esconômica e agregam valo nutricional, porém, ainda são poucos os estudos experimentais com objetivo de identificar o efeitos benéficos da ingestão de frutos nativos no organismo humano, a partir de uma especificação mais precisa da quantidade da ingestão diária recomendada.

Ressalta-se ainda que os estudos encontrados utilizaram a polpa dos frutos, óleo ou extrato com processos de trituração simples, não considerando a desestabilidade ou perda de nutrientes com a elaboração de receitas a partir de processos mais complexos.

Diante disso, espera-se que a revisão sirva de base para a elaboração de estudos com análises mais precisas quanto ao potencional nutricional de espécies nativas, tendo seres humanos como população de estudo, bem como para estimular novas pesquisas sobre o tema afim de ampliar o conhecimento da população em relação os benefícios dos frutos nativos.

\section{Agradecimentos}

A Universidade Federal de Mato Grosso do Sul_UFMS pelo incentivo e apoio à pesquisa cietífica.

Ao Conselho Nacional de Desenvolvimento Científico e Tecnológico - CNPq, pela bolsa de Iniciação Científica.

Ao Grupo de Estudos e Pesquisas em Enfermagem Clínica (GEPEC/UFMS), pelas experiências no universo científico.

\section{Declaração} de interesse.

Os autores declaram não existir qualquer conflito

\section{Referências}

Abramovay, R. Moratória para os Cerrados - Elementos para uma estratégia de agricultura sustentável. Disponível em: http: //www.econ.fea.usp.br/abramovay/outros_trabalhos/1999/ Moratoria_para_os_cerrados.pdf.

Amaral FP. Estudo das características físico-químicas dos óleos da amêndoa e polpa da macaúba [Acrocomia aculeata (Jacq.) Lodd. ex Mart], 2007 [Dissertação de Mestrado]. Universidade Estadual Paulista -UNESP. São Paulo/SP. Disponível em: $<$ https://repositorio.unesp.br/handle/11449/90468>. Acesso em: 03 out. 2019.

Almeida SP, SILVA JA. Oferta alimentar e componentes nutricionais de plantas nativas do cerrado. Embrapa Cerrados-Outras publicações técnicas. Ed:INFOTECAE.São Paulo,

1995. 
Ávidos MFD, Ferreira, LT. Frutos do Cerrado - Preservação gera muitos b frutos. Disponível em: $<$ http//www.biotecnologia.com.br/bio15/>. Acesso em: 19 de set. 2004

Allister EM, Borradaile NM.; Edwards JY, Huff M W.Inhibition of microsomal triglyceride transfer protein expression and apolipoprotein B100 secretion by the citrus flavonoid naringenin and by insulin involves activation of the mitogen-activated protein kinase pathway in hepatocytes. Diabetes Journals, 54,1676-1683, 2005. https://doi.org/10.2337/diabetes.54.6.1676.

Barbosa K.B.F, Costa NMB, Alfenas RDCG, Paula SO, Minim VPR., Bressan J. Estresse oxidativo: conceito, implicações e fatores modulatórios. Revista de nutrição, 23, 2010. https://doi.org/10.1590/S141552732010000400013.

Bento APN, Cominetti C, Simões Filho A, Naves, MMV. Baru almond improves lipid profile in mildly hypercholesterolemic subjects: A randomized, controlled, crossover study. Nutrition, metabolism and cardiovascular diseases, 24, 1330-1336, 2014. https://doi.org/10.1016/j.numecd.2014.07.002.

Coimbra MC. Caracterização dos frutos e dos óleos extraídos da polpa e amêndoa de guariroba (Syagrus oleracea), Jerivá (Syagrus romanzoffiana) e macaúba (Acromia aculeata), 2010. [Dissertação de Mestrado].Universidade Estadual Paulista - UNESP. São Paulo/MS.Disponível em: https://repositorio.unesp.br/handle/11449/88418.

Embrapa Cerrados. Produtos que poderão ser explorados comercialmente. 2006. Disponível em: <http://www.embrapa.br.htm>. Acesso em: 01 out. 2019 .

Hanke D. Zahradka P. Mohankumar SK, Clark JL, Taylor CG. A diet high in $\alpha$-linolenic acid and monounsaturated fatty acids attenuates hepatic steatosis and alters hepatic phospholipid fatty acid profile in diet-induced obese rats. Prostaglandins, Leukotrienes and Essential Fatty Acids, 89, 391-401, 2013. https://doi.org/10.1016/j.plefa.2013.09.009.

Jadad AR, Moore RA, Carroll D, Jenkinson C, Reynolds DJM, Gavaghan DJ, McQuay HJ. Assessing the quality of reports of randomized clinical trials: is blinding necessary?. Controlled clinical trials, 17, 1-12, 1996. https://doi.org/10.1016/0197-2456(95)00134-4

Kaur C, Kapoor HC. Antioxidants in fruits and vegetables-the millennium's health. International journal of food science \& technology, 36, 703-725, 2001.

Luzia, D. M. M. Propriedades funcionais de óleos extraídos de sementes de frutos do cerrado brasileiro, 2012 [Tese de Doutorado]. Universidade Estadual Paulista - UNESP. São José do Rio Preto/SP. Disponível em:< https://repositorio.unesp.br/handle/11449/100896Acesso em: 07 out. 2019 .

March JH. Levels of evidence. Oxford CEBM. 2009. Disponível em:https://www.cebm.net/wpcontent/uploads/2014/06/C EBM-Levels-of-Evidence-2.1.pdf. [acesso em: $20 \mathrm{de}$ agosto de 2018.

Marin AM, Siqueira EM, Arruda SF. Minerals, phytic acid and tannin contents of 18 fruits from the Brazilian savanna. International Journal of Food Sciences and Nutrition, 60 ,

2009.
Moreira AJ, Fraga C, Alonso M, Collado PS, Zetller C, Marroni C, González-Gallego J. Quercetin prevents oxidative stress and NF- $\mathrm{BB}$ activation in gastric mucosa of portal hypertensive rats. Biochemical pharmacology, 68, 1939194, 2004. https://doi.org/10.1016/j.bcp.2004.07.016

Nunes ÂA, Buccini DF, Jaques JA, Portugal LC, Guimarães RC, Favaro SP, Carvalho CM. Effect of Acrocomia aculeata kernel oil on adiposity in type 2 diabetic rats. Plant foods for human nutrition, 73, 61-67, 2018. https://doi.org/10.1007/s11130-017-0648-8.

Okuda T. Systematics and health effects of chemically distinct tannins in medicinal plants. Phytochemistry, 66, 20122031, 2005. https://doi.org/10.1016/j.phytochem.2005.04.023.

Olivera DL. Viabilidade econômica de algumas espécies medicinais nativas do cerrado. Revista EVS-Revista de Ciências Ambientais e Saúde, 38, 301-332, 2011.

Olefsky JM, Glass CK. Macrophages, inflammation, and insulin resistance. Аnnu Rev Physiol, 72, 219-46, 2010. https://doi.org/10.1146/annurev-physiol-021909-135846

Orsi PR, Bonamin F, Severi JA, Santos R C,Vilegas W, HirumaLima CA, Di Stasi LC. Hymenaea stigonocarpa Mart. ex Hayne: A Brazilian medicinal plant with gastric and duodenal anti-ulcer and antidiarrheal effects in experimental rodent models. Journal of Ethnopharmacology, 143, 81-90, 2012.

Reis MA, Novaes RD, Baggio SR, Viana ALM, Salles BCC, Duarte SMDS, Paula FBDA. Hepatoprotective and Antioxidant Activities of Oil from Baru Almonds (Dipteryx alata Vog.) in a Preclinical Model of Lipotoxicity and Dyslipidemia. Evidence-Based Complementary and Alternative Medicine, 2018. https://10.1155 / 2018/8376081.

Romero ABD, Martins MDC, Nunes PHM, Ferreira NRT, Brito AKDS, Cunha PFMD, Araújo EM. In vitro and in vivo antioxidant activity of Buriti fruit (Mauritia flexuosa L. f) / La actividad antioxidante in vitro e in vivo de la fruta burití (Mauritia flexuosa L. f). Nutricion Hospitalaeira, $32 \quad$ 2153-2161, 2015. http://dx.doi.org/10.3305/nh.2015.32.5.9603

Rodrigues ET. A influência dos frutos do Cerrado na diversificação da gastronomia. UnB, Brasília, 2004.

Rajendran P, Nandakumar N, Rengarajan T, Palaniswami R, Gnanadhas EN, Lakshminarasaiah U, Nishigaki I. Antioxidants and human diseases. Clinica chimica acta, 436, 332-347, 2014 https://doi.org/10.1016/j.cca.2014.06.004

Sloan AE. Top trends to watch and work on for the millennium. Food Technology, 53, 1999.

Silva CM. Potencialidades do Cerrado: Conhecer para proteger.2011.

Soares CB, Hoga LAK, Peduzzi MS, Carine YT, Silva DRAD. Revisão integrativa: conceitos e métodos utilizados na enfermagem. Revista da Escola de Enfermagem da USP, 48, 335-345, 2014. https://dx.doi.org/10.1590/S0080- 


\section{0}

Souza MT, Silva MD, Carvalho, R. Revisão integrativa: o que é e como fazer. Revista Einstein, 8, 102-106, 2010. https://dx.doi.org/10.1590/S008062342014000020 00020

Sundaram R, Naresh R, Shanti P, Sachdadnandan P. Modulatory effect of green tea extract on hepatic key enzymes ofglucose metabolism in streptozotocin and high fat diet induceddiabetic rats. Phytomedicine, 20, 577-584, 2013. https://doi.org/10.1016/j.phymed.2013.01.006.

Silva M R, Silva MS, Martins KA, Borges S. Utilização tecnológica dos frutos de jatobá-do-cerrado e de jatobáda-mata na elaboração de biscoitos fontes de fibra alimentar e isentos de açúcares. Ciência e Tecnologia de Alimentos, 21, 176-182, 2001.

Silva DB, Sillva JA, Junueira NTV, Andrade LRM. Frutas do Cerrado. Brasília, DF: Embrapa Informação Tecnológica. 2001; 178 p A.

Togashi M, Sgarbieri VC. Caracterização química parcial do fruto do baru (Dipteryx alata, Vog.). Ciência e Tecnologia de Alimentos, 141, 85-95, 1993.

Tomás BFA, Espín JC. Phenolic compounds and related enzymes as determinants of quality in fruits and vegetables. Journal of the Science of Food and Agriculture, 81, p.853-876, 2001. https://doi.org/10.1002/jsfa.885

Veloso C, Oliveira MC, Costa OC, Rodrigues VG, Giiusti Paiva A, Teixeira M, Castro PA. Hydroethanolic extract of Pyrostegia venusta (Ker Gawl.) Miers flowers improves inflammatory and metabolic dysfunction induced by high-refined carbohydrate diet. Journal of ethnopharmacology, 151, p.722-728, 2014. https://doi.org/10.1016/j.jep.2013.11.046.

Vieira, RF et al. Embrapa Informação Tecnológica Frutas nativas da região Centro-Oeste do Brasil, 2010. Disponível em:https://www.researchgate.net/profile/Josue_Junior/p ublication/265250187_Araca/links/54062bcc0cf2c48563 b248f1/Araca.pdf. Acessado em: 23/06/2018.

Wach A, Pryrzynska K, Biesaga M. Quercetin content in some food and herbal samples. Food Chemistry, 100, p. 699704 , 2007. https://doi.org/10.1016/j.foodchem.2005.10.028

Yuyama LK, Yonekura L, Aguiar JP, Sousa RF. Biodisponibilidade dos carotenoides do Buriti (Mauritia flexuosa L.) em ratos. Acta Amazonica, 28, 409-409, 1998. 